\title{
Fulminantes Rechtsherzversagen bei pulmonaler mikroskopischer Tumorzellembolie - eine seltene Differenzialdiagnose
}

\section{S. Yilmaz ${ }^{1}$ \\ J. Galle ${ }^{2}$ \\ L. Welker ${ }^{1}$ \\ G. Kanzow ${ }^{1}$}

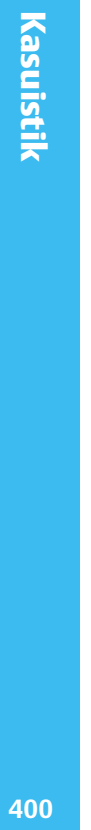

\author{
Right Heart Failure Due to Pulmonary Tumor Microembolism - \\ a Rare Differential Diagnosis
}

\section{Zusammenfassung}

Wir berichten über einen 69-jährigen Patienten, der mit progredienter Dyspnoe und Hämoptysen unter dem Verdacht auf ein Bronchialkarzinom stationär eingewiesen wurde. Der Patient verstarb am 12. Behandlungstag unter dem klinischen Verdacht auf das Vorliegen einer fulminanten Lungenembolie an den Folgen eines Rechtsherzversagens. Die Autopsie erbrachte den Nachweis von disseminierten mikroskopischen pulmonalen Tumorzellembolien auf dem Boden eines bronchogenen Adenokarzinoms des linken Oberlappens mit Befall der mediastinalen Lymphknoten. Hinweise auf eine venöse Thrombembolie ergaben sich nicht. Wenn bei Tumorpatienten mit Dyspnoe das Röntgenbild die Symptomatik nicht ausreichend erklärt, sollte frühzeitig an das Vorliegen von Tumorembolien gedacht werden, um im Zweifelsfalle eine belastende Diagnostik oder falsche Therapie vermeiden zu können.

\section{Abstract}

A 69-year-old man presented with progressive dyspnea, hemoptysis and fatigue for the previous 4 weeks. Chest radiograph and CT-scan were suggestive of lung cancer. The patient's condition worsened and within in few days he developed respiratory failure and acute cor pulmonale. Despite intensive care measures directed at suspected lung thromboembolism, the patient died on day 12 . Autopsy revealed disseminated obstruction of small pulmonary arteries by microscopic tumor emboli. No signs of venous thromboembolism were found. Pulmonary tumor microembolism should be considered whenever a patient with malignancy presents with unexplained progressive dyspnea or pulmonary hypertension.

\section{Falldarstellung}

Ein 69-jähriger ehemaliger Molkereiarbeiter wurde uns wegen einer seit vier Wochen zunehmenden Belastungsdyspnoe mit Leistungsabfall und seit drei Tagen bestehenden Hämoptysen (ca. $100 \mathrm{ml} / \mathrm{Tag}$ ) unter dem Verdacht auf das Vorliegen eines
Bronchialkarzinoms zugewiesen. Ernsthafte Vorkrankheiten ließen sich anamnestisch nicht erfragen. Keine regelmäßige Medikamenteneinnahme, keine Angabe von Fieber, Nachtschweiß oder Ödemen. Der Patient berichtete einen langjährigen inhalativen Nikotinkonsum bis zum Aufnahmetag (kumulativ 50 packyears) und einen täglichen Konsum von 3 Flaschen Bier.

Institutsangaben

${ }^{1}$ Krankenhaus Großhansdorf, Zentrum für Pneumologie und Thoraxchirurgie,

(Ärztlicher Direktor: Prof. Dr. med. H. Magnussen), Großhansdorf

${ }^{2}$ Forschungszentrum Borstel, Pathologie (Direktor: Prof. Dr. Dr. med. E. Vollmer), Borstel

Widmung

Herrn Professor Dr. H. Magnussen zum 60. Geburtstag gewidmet.

Korrespondenzadresse

Dr. Georg Kanzow · Krankenhaus Großhansdorf · Zentrum für Pneumologie und Thoraxchirurgie Wöhrendamm $80 \cdot 22927$ Großhansdorf

Eingang: 31. März 2004 · Angenommen: 19. April 2004

Bibliografie

Pneumologie 2004; 58: 400-403 • (c) Georg Thieme Verlag KG Stuttgart · New York

DOI $10.1055 / \mathrm{s}-2004-818458$

ISSN 0934-8387 
Bei der körperlichen Untersuchung zeigte sich ein leptosomer Patient in reduziertem Allgemeinzustand mit Dyspnoe bereits beim An- und Auskleiden. Bis auf eine Blässe der Haut und der einsehbaren Schleimhäute war der weitere Untersuchungsbefund unauffällig.

In der Laboruntersuchung fand sich eine makrozytäre Anämie (Hämoglobin 9,1 g/dl, MCV $100 \mathrm{fl}$ ). Das C-reaktive Protein betrug $7,9 \mathrm{mg} / \mathrm{l}$ (normal $<5 \mathrm{mg} / \mathrm{l}$ ), die Serum-Laktatdehydrogenase $501 \mathrm{U} / \mathrm{l}$ (normal 135-225 U/l), das Serum-Bilirubin 1,8 mg/dl (normal $<1,0 \mathrm{mg} / \mathrm{dl}$ ). Die übrigen Laborparameter waren unauffällig. In der Blutgasanalyse zeigte sich eine schwergradige respiratorische Partialinsuffizienz $\left(\mathrm{pO}_{2} 53 \mathrm{mmHg}, \mathrm{pCO}_{2} 30 \mathrm{mmHg}\right)$, die durch Gabe von 2 1/min Sauerstoff über eine Nasensonde ausgeglichen werden konnte. Der Tuberkulin-Stempeltest war unauffällig.

In der Lungenfunktionsprüfung fand sich eine leichtgradige obstruktive Ventilationsstörung (VC 4,11, entsprechend $85 \%$ des Sollwertes, $\mathrm{FEV}_{1} 2,4 \mathrm{l}$, entsprechend $68 \%$ des Sollwertes, $\mathrm{FEV}_{1} / \mathrm{VC}$ $59 \%$ ). Wegen der hochgradigen Dyspnoe konnte die Diffusionskapazität nicht gemessen werden. Im Röntgenbild der Thoraxorgane (Abb. 1) stellte sich ein etwa $3 \mathrm{~cm}$ großes, unscharf begrenztes Infiltrat in Projektion auf den linken Lungenoberlappen dar. Die Lungenhili wirkten beidseits verplumpt. Das Herz war unauffällig konfiguriert. Im von auswärts mitgebrachten Computertomogramm des Thorax sah man einen tumorverdächtigen Knoten mit streifigen Ausläufern im linken Oberlappen. Die mediastinalen und hilären Lymphknoten waren vergrößert (Abb. 2). Hinweise auf das Vorliegen einer Lymphangiosis carcinomatosa oder von Lungenembolien ergaben sich nicht.

Bei der Bronchoskopie fand sich eine leichte Blutung aus dem rechten Oberlappenbronchus, der endobronchiale Befund war ansonsten unauffällig. Im Bronchialsekret und im perbronchialen Punktat eines subkarinalen Lymphknotens waren keine Tumorzellen nachweisbar, die mikroskopische Untersuchung auf Mykobakterien war unauffällig. Wegen anhaltender Hämoptysen wurde eine Ösophagogastroduodenoskopie durchgeführt, die keinen auffälligen Befund zeigte. Bei der endosonographisch gesteuerten Punktion eines rechts-mediastinalen Lymphknotens wurden dann Einzelzellen eines malignen Tumors nachgewiesen, die nicht genau zugeordnet werden konnten. Differenzialdiagnostisch wurde auch an ein malignes Lymphom gedacht. Die Beckenkammbiopsie zeigte entzündungs- und tumorfreies Knochenmark. Als nächster diagnostischer Schritt war eine Mediastinoskopie geplant.

Der weitere Verlauf zeichnete sich durch zunehmende Dyspnoe und körperliche Schwäche aus. Die Hämoptysen persistierten, sodass Bluttransfusionen erforderlich wurden. Es entwickelten sich Zeichen einer Rechtsherzdekompensation mit beidseitigen Unterschenkelödemen und einer Lebervenenstauung. Der Patient verstarb am 12. Behandlungstag trotz intensivmedizinischer Maßnahmen unter dem klinischen Verdacht einer fulminanten Lungenembolie.

Bei der Obduktion fand sich in der linken Oberlappenspitze ein $3,2 \mathrm{~cm}$ großes, subpleural gelegenes, niedrig ausdifferenziertes Adenokarzinom der Lunge mit teilweise stark dissoziierten Ein-

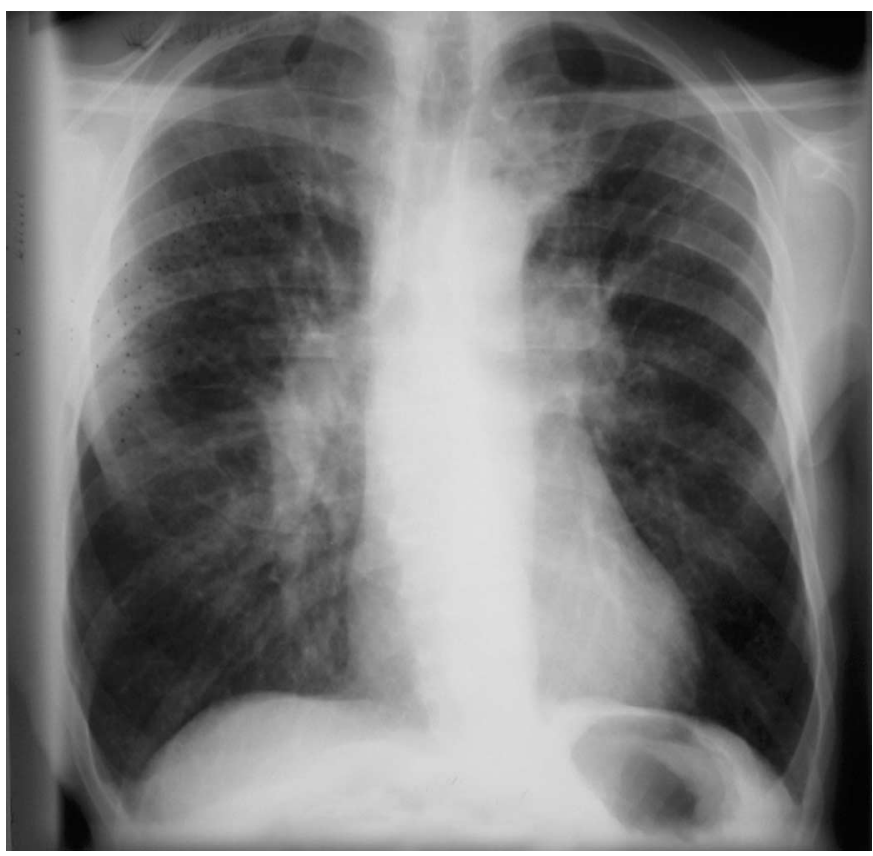

Abb. 1 Röntgenbild der Thoraxorgane mit Verschattung im linken Oberfeld und prominenten Lungenhili.

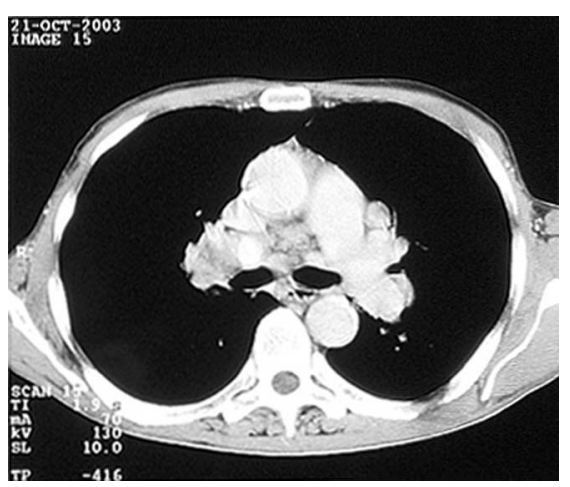

Abb. 2 Das Computertomogramm (Weichteilfenster) zeigt vergrößerte mediastinale Lymphknoten.

zelzellverbänden sowie peribronchialen und paratrachealen Lymphknotenmetastasen. Weiterhin fielen in beiden Lungen in mittel- und kleinkalibrigen arteriellen und venösen Pulmonalgefäßen disseminierte Tumorembolien auf, die in den Arterien vielfach von einer verschließenden Intimafibrose begleitet waren (Abb. 3a/b). Durch die Okklusion der pulmonalen Strombahn kam es zu einem finalen Rechtsherzversagen. Eine venöse Thrombembolie konnte nicht nachgewiesen werden.

\section{Diskussion}

Unser Patient wurde unter dem Verdacht auf ein Bronchialkarzinom eingewiesen. Zytologisch konnten im Punktat aus einem mediastinalen Lymphknoten Tumorzellen nachgewiesen werden. Innerhalb weniger Tage kam es zu einer progredienten Rechtsherzdekompensation, und der Patient verstarb unter dem Verdacht auf eine Lungenembolie. Durch die Autopsie konnte ein bronchogenes Adenokarzinom nachgewiesen werden, als Todesursache fanden sich pulmonale mikroskopische Tumorzellembolien in beiden Lungen. Auffällig waren im Primärtumor stark dis- 

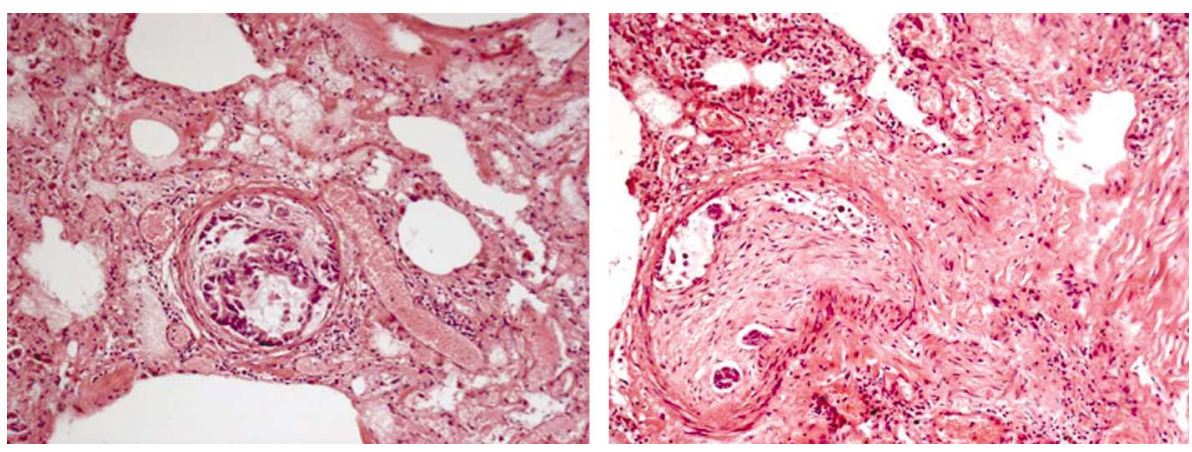

Abb. 3 a und b Arterielle Pulmonalgefäße mit Tumorzellemboli. (a) Geringgradige Intimareaktion, (b) deutliche Intimafibrose mit subtotaler Gefäßobliteration (HE-Färbung).

soziierte Tumorzellverbände. Dieser Befund spricht möglicherweise für eine stark herabgesetzte Zelladhäsion, hierdurch könnte sich die außergewöhnlich massive Aussaat im Gefäßsystem erklären.

Pulmonale Tumorzellembolien werden zu Lebzeiten selten diagnostiziert $[1,2]$. Selbst bei Patienten mit gesicherter Tumorerkrankung wird die korrekte Diagnose nur in 6\% der Fälle intra vitam gestellt [3]. Aus Sektionsstatistiken geht jedoch hervor, dass die Häufigkeit derartiger Embolien bei Patienten mit soliden Neoplasmen (Vorhofmyxome, Mamma-, Leber-, Magen-, Pankreas-, und Nierenkarzinom, Chorionkarzinom u. a.) bei 2,6-26\% liegt und in $1-8 \%$ als Todesursache angesehen wird [4-6]. Für das nichtkleinzellige Bronchialkarzinom wird die Inzidenz nach einer Autopsiestudie mit 10\% angegeben [7]. Pathologisch ist die pulmonale Tumorzellembolie charakterisiert durch den Nachweis von einzelnen Tumorzellen oder Tumorzellverbänden innerhalb der pulmonalarteriellen Strombahn einschließlich der Alveolarkapillaren. Pathogenetisch werden fünf Formen der Embolisation unterschieden $[4,8]$ :

- große Tumorzellembolie mit Verschluss von proximalen Lungenarterienabschnitten oder von Segmentarterien

- generalisierte Tumorinfiltration der Lymphgefäße

- mikroskopische Tumorzellembolien mit Befall der kleinen Pulmonalgefäße einschließlich der Arteriolen

- Kombination von 1, 2 und 3

- diffuse Embolisation der Alveolarkapillaren.

Tumorzellembolien treten in der Regel klinisch unter den Zeichen eines subakuten oder akuten Cor pulmonale in Erscheinung. Große Embolien, verursacht durch abgelöste Tumorzapfen, werden insbesondere bei Nierenzellkarzinomen oder Wilms-Tumoren angetroffen und führen akut zu einer Rechtsherzdekompensation wie bei einer fulminanten Thrombembolie. Bei der häufigeren mikroskopischen Tumorzellembolie kommt es hingegen meist zu einer schubweisen, subakuten Rechtsherzbelastung $[3,9]$. Histologisch zeigt sich nicht immer ein kompletter Verschluss des Gefäßlumens durch Tumorzellen. Es werden jedoch durch die Embolisation komplexe Prozesse in Gang gesetzt, die neben einer Gerinnungsaktivierung mit Ausbildung von Mikrothromben auch eine entzündliche Gefäßreaktion sowie eine irreversible Intimafibrose der Gefäßwand umfassen [4,6] (Abb.3). Folge ist eine zunehmende pulmonalarterielle Druckerhöhung. Mikrovaskuläre Embolien sind wahrscheinlich auch die Ursache der pulmonalen Lymphangiosis carcinomatosa [10]. Weniger als $1 \%$ aller Tumorzellen, die in die Blutzirkulation gelangen, überstehen die Lungenpassage. Die meisten Zellen werden durch die
Scherkräfte in der Mikrozirkulation oder durch lokale Immunmechanismen zerstört. Tumorzellen können jedoch die Wände von Lungenkapillaren durchdringen und auf diese Weise in die Lymphbahnen gelangen. Ein weiterer Metastasierungsweg führt retrograd von tumorbefallenen hilären Lymphknoten in die pulmonalen Lymphbahnen [11].

\section{Diagnosesicherung}

Die klinischen Symptome sind vielfältig. Am häufigsten findet sich progrediente Dyspnoe, die sich akut, über wenige Tage oder innerhalb weniger Monate entwickelt. Typisch sind Husten, Pleuraschmerz und Hämoptysen. Weiterhin können Allgemeinbeschwerden im Sinne einer tumorassoziierten B-Symptomatik vorhanden sein. Schwierigkeiten bei der Diagnostik bereitet die klinische Ähnlichkeit mit akuten Thrombembolien, die bekanntermaßen bei malignen Erkrankungen gehäuft auftreten. Zur Abgrenzung kann das Fehlen einer Emboliequelle (z. B. in den tiefen Beinvenen) und das fehlende Ansprechen auf eine medikamentöse Antikoagulation genutzt werden. Gelegentlich ist, wie bei unserem Patienten, die Tumorzellembolie die erste klinische Manifestation des zu Grunde liegenden Malignoms [12].

Die Blutgasanalyse zeigt typischerweise eine schwergradige respiratorische Partialinsuffizienz. Im EKG und in der Echokardiographie können sich Hinweise für eine Rechtsherzbelastung finden. Die konventionellen Röntgenaufnahmen sind in vielen Fällen unauffällig. Es können jedoch Zeichen der pulmonalen Hypertonie, Pleuraergüsse sowie lokalisierte oder disseminierte Lungeninfiltrate bestehen. Im Lungenperfusionsszintigramm finden sich typischerweise multiple subsegmentale Perfusionsausfälle [13]. Auch wenn dieser Befund als charakteristisch für das Vorliegen von mikroskopischen Tumorzellembolien gilt, ist er wenig spezifisch, da pulmonale Vaskulitiden, die primäre pulmonale Hypertonie und Fettembolien zu einem ähnlichen Bild führen können. Die Pulmonalisangiographie dient in erster Linie dem Ausschluss einer venösen Thrombembolie. Die durch Tumormikroembolien verschlossenen Arteriolen und Kapillaren können mit dieser Methode aufgrund der begrenzten Bildauflösung nicht erfasst werden. Gelegentlich finden sich jedoch eine verzögerte Füllung von Segmentarterien, eine vermehrte Schlängelung von Gefäßen der dritten bis fünften Generation und subsegmentale, 1-2 mm messende Füllungsdefekte [14]. Ähnlich wie die Angiographie ist auch die Computertomographie aufgrund der begrenzten Auflösung in der Regel nicht in der Lage, tumorbedingte mikroembolische Gefäßverschlüsse darzustellen. Hinweisend sind dilatierte und perlschnurartig deformierte periphere Pulmonalarterien. Hiervon sind differenzialdiagnos- 
tisch die perlschnurartig verdickten Septen bei Lymphangiosis carcinomatosa abzugrenzen [15]. Die Rechtsherzkatheter-Untersuchung weist in der Regel pulmonale Mitteldrucke von 50-100 mmHg nach. Der pulmonalkapilläre Verschlussdruck ist normal [16]. Über den in Wedge-Position geblockten Pulmonaliskatheter kann darüber hinaus Blut aus der Lungenperipherie für zytologische Untersuchungen gewonnen werden. Bei Patienten mit mikroskopischen Tumorembolien können mit dieser Methode mit hoher Sensitivität Tumorzellen nachgewiesen werden [17]. Die offene oder videoassistierte thorakoskopische Lungenbiopsie gelten als Goldstandard in der Diagnostik. Die transbronchiale Lungenbiopsie birgt wegen der in der Regel bestehenden schwergradigen pulmonalarteriellen Hypertonie ein hohes Blutungsrisiko.

\section{Therapie und Prognose}

Die therapeutischen Möglichkeiten sind begrenzt. Bei chemosensiblen Tumoren wird die Überlebenszeit möglicherweise durch eine adäquate antineoplastische Chemotherapie oder Hormonbehandlung verlängert, so z. B. bei Chorionkarzinom, Brustkrebs oder Prostatakarzinom; kontrollierte Studien für diese spezielle Patientengruppe existieren jedoch nicht. In seltenen Fällen führt die komplette Resektion des Primärtumors zu einer Remission [11]. Große Tumoremboli können gelegentlich erfolgreich chirurgisch entfernt werden $[18,19]$. Bei den meisten Patienten muss sich die Behandlung allerdings auf supportive Maßnahmen wie Sauerstoffgabe, Drainage von Pleuraergüssen und die Gabe von Opiaten beschränken. Auch systemische Kortikosteroide können kurzfristig zu einer Symptomlinderung führen, möglicherweise über eine Suppression der tumorinduzierten, entzündlichen Gefäßreaktion. Eine medikamentöse Antikoagulation besitzt keinen Stellenwert in der Behandlung [12].

\section{Schlussfolgerung}

Bei Patienten mit einer malignen Neoplasie sollte bei zunächst unerklärter, progredienter Dyspnoe oder Zeichen einer pulmonalarteriellen Hypertonie an das Vorliegen einer pulmonalen Tumorzellembolie gedacht werden, insbesondere dann, wenn das Röntgenbild der Thoraxorgane die Beschwerden nicht erklärt. Bei Vorliegen einer respiratorischen Partialinsuffizienz macht ein typisches Muster in der Lungenperfusionsszintigraphie die Diagnose wahrscheinlich. Die thorakale Computertomographie kann durch den Nachweis von perlschnurartig veränderten peripheren Pulmonalarterien die Verdachtsdiagnose unterstützen. Über den Rechtsherzkatheter kann Blut aus der Pulmonalisstrombahn für eine zytologische Untersuchung gewonnen werden. Der Nachweis von Tumorzellen (Cave: Verwechslung mit Megakaryozyten!) gilt als beweisend. Goldstandard in der
Diagnostik ist die offene oder videoassistierte thorakoskopische Lungenbiopsie. Eine zielgerichtete Diagnostik kann dazu beitragen, dem Patienten unnötige Behandlungen wie Antikoagulation, Fibrinolyse oder den Einsatz eines Vena-cava-Schirms zu ersparen.

\section{Literatur}

${ }^{1}$ Schriner RW, Ryu JH, Edwards WD. Microscopic pulmonary tumor embolism causing subacute cor pulmonale: a difficult antemortem diagnosis. Mayo Clin Proc 1991; 66: $143-148$

2 Roberts KE, Hamele-Bena D, Saqi A et al. Pulmonary tumor embolism: a review of literature. Am J Med 2003; 115: 228-232

${ }^{3}$ Goldhaber SZ, Dricker E, Buring JE et al. Clinical suspicion of autopsyproven thrombotic and tumor pulmonary embolism in cancer patients. Amer Heart J 1987; 114: 1432 - 1435

${ }^{4}$ Kane RD, Hawkins HK, Miller JA et al. Microscopic pulmonary tumor emboli associated with dyspnea. Cancer 1975; 36: 1473-1482

${ }^{5}$ Veinot JP, Ford SE, Price RG. Subacute cor pulmonale due to tumor embolization. Arch Pathol Lab Med 1992; 116: 131 -134

${ }^{6}$ Soares FA, Pinto AP, Landell GA et al. Pulmonary tumor embolism to arterial vessels and carcinomatous lymphangitis. A comparative clinopathological study. Arch Pathol Lab Med 1993; 117: 827-831

7 Gonzalez-Vitale JC, Garcia-Bunuel R. Pulmonary tumor emboli and cor pulmonale in primary cancer of the lung. Cancer 1976; 38: 2105- 2110

8 Abbondanzo SL, Klappenbach RS, Tsou E. Tumor cell embolism to pulmonary alveolar capillaries. Cause of sudden cor pulmonale. Arch Pathol Lab Med 1986; 110: 1197 - 1198

${ }^{9}$ Fanta CH, Compton CC. Microscopic tumor emboli to the lungs: a hidden cause of dyspnea and pulmonary hypertension. Thorax 1979; 43: $794-795$

${ }^{10}$ King MB, Harmon KR. Unusual forms of pulmonary embolism. Clin Chest Med 1994; 15: $561-580$

${ }^{11}$ Mehrishi S, Awan A, Mehrishi A et al. Pulmonary tumor microembolism. Hosp Physician 2004; 40: 23 - 30

${ }^{12}$ Montero A, Vidaller A, Mitjavila F et al. Microscopic pulmonary tumoral embolism and subacute cor pulmonale as the first clinical signs of cancer. Acta Oncol 1999; 38: 1116 - 1118

${ }^{13}$ Sostman HD, Brown M, Toole A. Perfusion scan in pulmonary vascular/ lymphangitic carcinomatosis: the segmental contour pattern. Am J Roentgenol 1981; 137: 1072 - 1074

${ }^{14}$ Margolis ML, Jarrell BE. Pulmonary tumor microembolism. South Med J 1985; 78: 757 - 758

${ }^{15}$ Shepard JA, Moore EH, Templeton PA et al. Pulmonary intravascular tumor emboli: dilated and beaded peripheral pulmonary arteries at CT. Radiology 1993; 187: 797-801

${ }^{16}$ Chan CK, Hutcheon MA, Hyland RA. Pulmonary tumor embolism: a critical review of clinic, imaging, and hemodynamic features. J Thorac Imaging 1987; 2: 4-14

${ }^{17}$ Masson RG, Ruggieri J. Pulmonary microvascular cytology. A new diagnostic application of the pulmonary artery catheter. Chest 1985; 88: $908-914$

${ }^{18}$ Keenan DJ, Morton P, O’Kane HO. Right atrial myxoma and pulmonary embolism. Rational basis for investigation and treatment. Br Heart J 1982; 48: 510-512

19 Isringhaus H, Naber M, Kopper B. Successful treatment of tumor embolism of a hypernephroma with complete occlusion of the left pulmonary artery. Thorac Cardiovasc Surg 1987; 35: 65-66 